\title{
Excitations in one-dimensional lattices with traps: Exact results and simulations
}

\author{
Francisco Domínguez-Adame ${ }^{\mathrm{a}}$, Miguel Angel Rodríguez ${ }^{\mathrm{b}}$, Angel Sánchez ${ }^{\mathrm{c}}$ \\ aepartamento de Física de Materiales and Grupo Interdisciplinar de Sistemas Complicados, Universidad Complutense, \\ E-28040 Madrid, Spain \\ b Instituto de Física de Cantabria, CSIC-UC, Universidad de Cantabria, E-39005 Santander, Spain \\ ${ }^{c}$ Departamento de Matemáticas and Grupo Interdisciplinar de Sistemas Complicados, Escuela Politécnica Superior, \\ Universidad Carlos III, E-2891I Leganés, Madrid, Spain
}

\begin{abstract}
We study motion and capture of incoherent excitons in one-dimensional lattices with randomly placed, perfectly absorbing traps. Large-scale numerical simulations of both the master equation and the random walk version of the problem disagree with the result for the survival fraction obtained long ago by Movaghar et al. [J. Stat. Phys. 27 (1982) 473]. We carry out exact calculations for this problem and find that the disagreement can be traced back to rare-event contributions to the analytical result which cannot be seen in simulations. We conclude that for practical purposes, i.e., for averages taken over any finite number of realizations, the only asymptotics observable is the one we have found in our simulations.
\end{abstract}

PACS: 71.35.+z; 05.60.+w; 02.60.Cb; 61.43.-j

\section{Introduction}

Models of diffusion and trapping of excitations in one-dimensional (1D) random lattices are of interest in different physical situations, which include sensitized luminescence experiments in molecular aggregates, polymers in random media, and diffusion-limited reactions (see Ref. [1] and references therein). Whenever the trapping process (for example of an exciton) is rapid in comparison with the characteristic time scale associated with the diffusion mechanism of motion (of the excitons), the diffusion process becomes the most important factor in determining the kinetics of the system [2]. In this context, random walks on lattices with randomly lo- cated traps have received a lot of attention in the past few years as suitable models of those phenomena. Numerous researchers devoted their attention to this problem in the seventies and early eighties. Thus, it was established that for traps randomly distributed in a $d$-dimensional Euclidean space, the asymptotic decay of the survival fraction of random walkers, $n(t)$, is given by a stretched exponential $n(t) \sim$ $\exp \left[-\alpha c^{2 /(d+2)} t^{d /(d+2)}\right]$, with $c$ the concentration of traps and $\alpha$ a dimension-dependent constant [3]. There are more detailed results for one dimension (see Refs. [4,5] and references therein), computing even the prefactor to the exponential dependence. To our knowledge, however, none of these results was ever compared to simulations. 
After the mid-eighties, the interest in this problem considerably decreased, as it was regarded as basically solved. In fact, even analytical solutions of the master equation for the exciton motion in the infinite chain with a single trap have been found by several authors [6-8]. Recently, however, a renewed interest in this question arose in connection to results on electron localization in disordered systems (see references in Ref. [9]), and diffusion of excitations in one-dimensional systems with traps placed at random but with some correlation among their position was studied, both in the classical [9] and the quantum case [10]. In Ref. [9], we carried out simulations to compare the correlated with the pure random case and the results were (in both cases) closer to $\exp \left(-A t^{1 / 2}\right)$ than to $\exp \left(-A t^{1 / 3}\right)$, the theoretical result predicted by several authors $[3,4,11]$. As the amount of computational work in Ref. [9] was not enough to make any definite claim on this question, we postponed its analysis until more data were available.

In this paper, we present results of a large scale simulation program intended to clarify this point. In Section 2 we report on the outcome of our numerical work, which can be summarized by saying that it was definitely established that the asymptotic regime was described with great accuracy by a functional dependence of the form $\exp \left(-A t^{1 / 2}\right)$. In view of this, we undertook the task of analytically studying the problem, to find out what was the reason for the discrepancy between the known results and our simulations. This we describe in full detail in Section 3, where we show how the key to understand the different exponents is found by a careful analysis. As we discuss below, our computations allow us to identify the origin of the disagreement with rare-event contributions to the analytical result which cannot be seen in simulations. Finally, in Section 4 we summarize our main conclusions.

\section{Model and simulations}

The basic equation governing the time evolution of the probability $P_{k}(t)$ to find a walker (or exciton) at site $k$ in a 1D lattice is the following master equation,

$$
\frac{\mathrm{d}}{\mathrm{d} t} P_{k}=\frac{D}{a^{2}}\left(P_{k+1}+P_{k-1}-2 P_{k}\right)-\gamma_{k} P_{k},
$$

where $a$ is the lattice spacing and $D$ is the diffusion coefficient, which is assumed to be constant over the whole lattice. Here $\gamma_{k}$ is the trapping rate, taken to be either infinite with probability $w$ or zero with probability $(1-w)$. In many physical situations of interest, and in others at sufficiently low temperature, traps are quenched, and then we will consider a static arrangement of traps. This master equation is solved assuming that the exciton is initially at arbitrary lattice site $k_{0}$, that is, $P_{k}(0)=\delta_{k k_{0}}$. Once this equation is solved, the survival fraction $n(t)=\sum_{k} P_{k}(t)$, which is the magnitude of interest in luminescence experiments as well as in other contexts, can be readily evaluated.

We have carried out numerical simulations by usual random-walk techniques. Our starting point is the master equation (1) with a given concentration of randomly distributed, perfectly absorbing traps. Numerical simulation proceeds as follows. We consider an exciton initially at site $k_{0}$ of the lattice. This site is chosen at random over the whole lattice. Let $\delta t$ be the time step and $\Delta \equiv D \delta t$. The exciton jumps to the neighboring left or right site with equal probability $\Delta$ if there is not any trap at its current position, whereas if there is a trap the exciton is trapped (i.e., it is annihilated). Periodic boundary conditions are used, so that there is no annihilation at the edges of the lattice. Once the exciton is annihilated, or the maximum time of the simulation is reached, a new exciton is started from another initial site $k_{0}$. Averages over an ensemble of excitons straightforwardly yields the survival fraction as a function of time, or any other quantity of interest. We have performed simulations with up to $5 \times 10^{4}$ excitons and $10^{5}$ time steps maximum per exciton, finding a very good agreement between simulations with these and smaller parameters. As a further test, we simulated several cases with $10^{7}$ excitons and $10^{6}$ time steps, finding always the same results. Besides, direct numerical integration of Eq. (1) with finite values of $\gamma_{k}$ [9] also agree with the outcome of the above described simulation procedure. In order to compare with previous analytical results [4], we have considered the high dilution limit, that is, the probability $w$ never exceeds the value 0.1 .

A typical example of the outcome of our simulations is shown in Fig. 1, where results for different values of $w=a$ are collected. In order to extract information about the time dependence of the survival fraction, we have plotted $\ln [-\ln n(t)]$ versus $\ln \left(a^{2} t\right)$. There- 


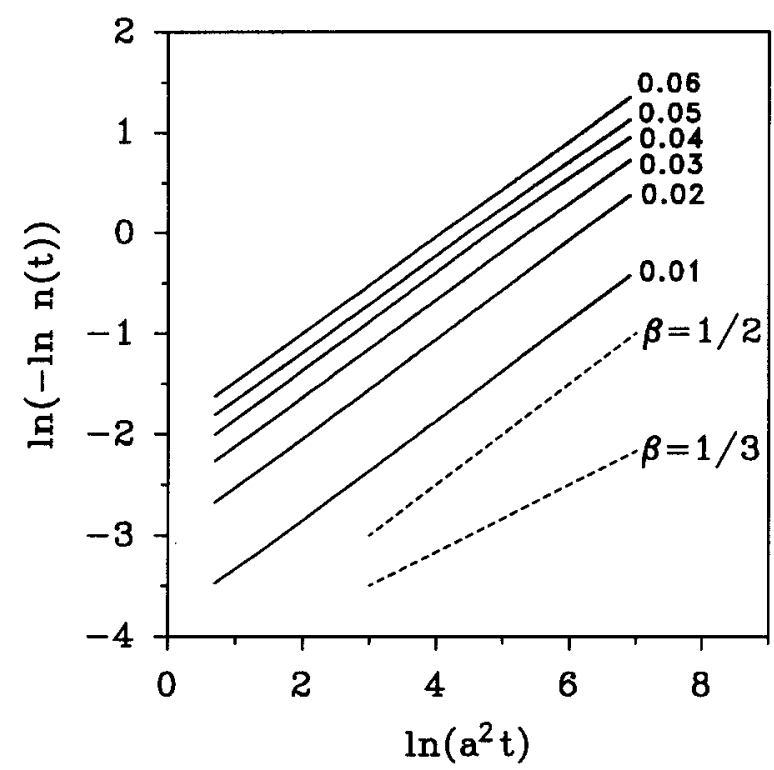

Fig. 1. Survival fraction of random walkers as a function of time for different values of the lattice spacing $a$ and probability $w$ (from bottom to top $w=a=0.01, \ldots, 0.06$ ). To extract the time dependence we plot $\ln [-\ln n(t)]$ versus $\ln \left(a^{2} t\right)$. Curves are well fitted by stretched exponentials $n(t) \sim \exp \left(-A t^{\beta}\right)$. For comparison, dashed lines indicate $\beta=1 / 2$ and $\beta=1 / 3$.

fore, the slope of those curves is simply the value of the exponent of the stretched exponential. In Fig. 1 parameters are $D=1$ and $\delta t=0.01$. Results comprise 25 ensemble averages per value of the probability $w$. Other choices of parameters give basically the same results. In all cases the results are very well described by stretched exponentials of the form $n(t) \sim$ $\exp \left(-A t^{\beta}\right)$, the exact value of the parameter $A$ being dependent on the probability $w$. However, the parameter $\beta$ is independent, within the numerical uncertainty, of $w$. The value of the exponent is $\beta \simeq 1 / 2$ in all cases we have studied, quite different from the theoretically predicted value $\beta=1 / 3$ in one dimension.

\section{Analytical results and discussion}

The exact calculation of the survival fraction is possible in the limit of perfect absorption since the exciton can only be captured by the nearest traps. Assuming that these traps are placed $l$ sites to the left and $m$ sites to the right of the initial position of the exciton, the survival fraction can be written as $n(t)=\sum_{l=1}^{\infty} \sum_{m=1}^{\infty} w(l, m) P_{-l, m}(t)$

Here $w(l, m)$ is the probability of having the already mentioned configuration of traps, which in the uniform case with probability $w$ reduces to $w(l, m)=$ $w^{l+m}(1-w)^{2}$, and $P_{-l, m}$ is the survival probability of a single particle starting at $k=0$ and with perfectly absorbing boundaries at $k=-l$ and $k=m$. This probability is calculated as $P_{-l, m}(t)=\sum_{k=-l}^{m} P_{k}(t)$, where $P_{k}(t)$ is the solution of the master equation (1) without the absorbing term $\left(\gamma_{k}=0\right)$ but with $P_{-l}(t)=$ $P_{m}(t)=0$ as boundary conditions. The direct calculation of $P_{k}(t)$ in the discrete case is quite complicated. However, in terms of the Laplace transform, $P_{-l, m}(s)$, the master equation becomes algebraic and can be easily solved. Thus, in the case of uniform distribution of traps, the Laplace transformed survival fraction $n(s)$ is readily calculated [4] but now the difficulty appears in the inversion to the time variable. In Ref. [4] a plausible but uncontrolled approximation has been developed. In this approximation the survival fraction, given by

$$
\begin{aligned}
& n(t)=\frac{2}{\pi^{2}} \sum_{l=0}^{\infty} \frac{1}{\left(l+\frac{1}{2}\right)^{2}} \\
& \times \int_{0}^{\infty} \mathrm{d} y y \exp \left(-y-\frac{\pi^{2} w^{2}(2 l+1)^{2} D t}{a^{2} y^{2}}\right),
\end{aligned}
$$

exhibits an asymptotic behavior as

$n(t) \sim \exp \left[-3\left(\frac{\pi^{2} w^{2} D t}{4 a^{2}}\right)^{1 / 3}\right]$.

Hence, this is the classical analytical result that one would expect to obtain in simulations. As it does not occur, first we have analyzed the above mentioned approximation. It is not difficult to check that this approximation coincides exactly with the continuum limit $a \rightarrow 0$, in which $c=w / a$ is the concentration of traps and the survival fraction becomes

$n(t)=\int_{0}^{\infty} \mathrm{d} y \int_{0}^{\infty} \mathrm{d} x c^{2} \exp [-c(x+y)] P_{-y, x}(t)$, 
$P_{-y, x}(t)$ being the corresponding survival probability given by $\int_{-y}^{x} \mathrm{~d} z P(z, t)$. The function $P(z, t)$ can be directly calculated from the classical diffusion equation $\partial_{t} P(z, t)=D \partial_{z z} P(z, t)$ with absorbing boundary conditions $P(-y, t)=P(x, t)=0$, obtaining

$$
\begin{gathered}
P(z, t)=\sum_{l=-\infty}^{\infty}\left(\frac{1}{x+y}\right) \cos \left(\frac{\pi(2 l+1)(y-x)}{2(x+y)}\right) \\
\quad \times \cos \left(\frac{\pi(2 l+1) z}{x+y}\right) \exp \left(-\frac{\pi^{2}(2 l+1)^{2} D t}{(x+y)^{2}}\right) .
\end{gathered}
$$

Substituting $P_{-y, x}(t)$, calculated from (5), into (4) we obtain (3a).

In view of the preceding discussion, expression (3b) from Ref. [4] should describe our simulations in the continuum limit. The continuum limit can be approximated in simulations taking values of parameters such that $D$ and $c$ remain constant when 1 $a \rightarrow 0$. However, as we have shown in the above section, instead of the expected result $\exp \left(-A t^{1 / 3}\right)$ we have always obtained the asymptotic behavior $\exp \left(-A t^{1 / 2}\right)$, and therefore the differences between the continuum and discrete versions of the problem are not responsible for the discrepancy. Hence, in order to pursue further the origin of this disagreement we have analyzed with more detail the physical meaning of the expression (3a). First, we introduce in our analysis the span $S$ as the length of the smallest interval that entirely contains the random walk. In terms of $S$ the survival fraction can be written, from (4), as $n(t)=\int_{0}^{\infty} \mathrm{d} S \exp (-S c) \theta_{S}(t)$, where $\theta_{S}(t)=\int_{0}^{S} \mathrm{~d} x \partial_{S} P_{-(S-x), x}(t)$ is the probability density of having a span $S$ in time $t$. Taking dimensionless times by setting $z=2 \pi^{2} c^{2} D t$ and measuring spans in units of the diffusion length with the change of variable $S=y z^{1 / 2}$ we have

$$
n(z)=\int_{0}^{\infty} \mathrm{d} y \exp \left(-y z^{1 / 2}\right) \theta(y),
$$

with

\footnotetext{
${ }^{1}$ In our simulations we take $D=c=1$. Plotting results of Fig. 1 as a function of $\ln t$ one finds that all curves overlap. This indicates that we are simulating correctly the limit $a \rightarrow 0$.
}

$$
\begin{gathered}
\theta(y)=\frac{8}{y^{3}} \sum_{l=0}^{\infty}\left(\frac{\pi^{2}(2 l+1)^{2}}{y^{2}}-1\right) \\
\times \exp \left(-\frac{\pi^{2}(2 l+1)^{2}}{2 y^{2}}\right) .
\end{gathered}
$$

The shape of this function, as can be seen in Ref. [12], is quite asymmetric. For small spans, $y<1$, the function has a tail of very low probability, $\theta(y) \sim$ $\exp \left(-\pi^{2} / 2 y^{2}\right)$ as $y \rightarrow 0$. Any event comprised in this tail cannot be reproduced in simulations and this is the reason for the disagreement between theory and simulations. The true asymptotic behavior of the survival fraction is dominated by these rare events since the maximum of the integrand in (6a) goes like $y_{\max } \sim$ $\pi^{2 / 3} z^{-1 / 6}$. An analytical treatment of $(6 a)$, for instance using a steepest descent method, gives the theoretical result, but any technique based on the occurrence of real events can lead to a very different behavior. To estimate this behavior we take into account that the integrand of (6a) is dominated by the low probability tail of $\theta$ when $y \ll y_{\max }$ and by the exponential term $\exp \left(-y z^{1 / 2}\right)$ when $y \gg y_{\max }$. On increasing time $y_{\max } \rightarrow 0$ and the asymptotic survival fraction is dominated by more and more rare events. Using a method able to take into account events with $y>y_{0}$, after a time $t \gg t_{0}=\pi^{4} / y_{0}^{6}$, the survival fraction can be estimated by

$n(z) \sim \exp \left(-y_{0} z^{1 / 2}\right)$.

Hence, the observed asymptotic behavior is reproduced. In simulations with $N$ realizations a rough estimation of $y_{0}$ would be $N^{-1}=\int_{0}^{y_{0}} \mathrm{~d} y \theta(y)$. Numerical analysis leads to $y_{0}^{2} \sim 1 / \log N$, which indicates the slow variation of the factor in the asymptotic behavior (7) with the number of realizations. To give an idea of this variation we have.calculated $y_{0}$ and the time in which the exponential behavior appears for 20,5000 , and 70000 realizations obtaining $y_{0}=0.974,0.658,0.589$ and $t_{0}=5.77,60.79,118.18$ respectively. Our simulations show also a slight decrease of the prefactor on the number of realizations, although this decrease is not quite as our rough estimation predicts. This discrepancy calls for a more detailed analysis of this specific point, which is out of the scope of the present work.

We can summarize the above analysis by saying that, in mathematical terms, the limits $N \rightarrow \infty$ and 
$t \rightarrow \infty$ do not commute at all. The asymptotic behavior calculated from simulations, in which one takes the temporal limit before the number of realizations going to infinity, is different from calculations by analytical methods, which are independent of the realizations of events. Nonetheless, in physics these events are present, this being the reason why we claim that $\exp \left(-A t^{1 / 2}\right)$ is the true (i.e., physically relevant) asymptotic behavior.

\section{Conclusions}

In this paper, we have reexamined the problem of excitation diffusion in lattices with perfectly absorbing traps to understand the discrepancy of the exact results in Ref. [4] with the simulations in Ref. [9]. After carrying out more simulations, which confirmed the result that the asymptotics of the survival fraction was $n(t) \sim \exp \left(-A t^{1 / 2}\right)$, we looked again at the previous analytical works. We verified that the expression given by Movaghar et al. [4], although derived through an uncontrollable assumption, is exact in the continuum limit $a \rightarrow 0$. Simulations with parameters progressively approaching this limit showed no sign of separation from the $\exp \left(-A t^{1 / 2}\right)$ behavior, meaning that the discrepancy between theory and numerics cannot be due to differences between the continuum and the discrete versions of the problem. Further insight into this question was provided by the reformulation of the calculations in terms of the span, which finally pinpointed the key to understanding the discrepancy, by revealing that there are events (in the small span limit) with vanishing probability and which simulations can by no means include. We also discussed how this difficulty can be interpreted in terms of the fact that the $N \rightarrow \infty$ and the $t \rightarrow \infty$ limits do not commute, thus leading to different analytical and numerical results.

We believe that our conclusions are important both theoretically and experimentally. From this last viewpoint, our finding that the decreasing of the survival fraction in simulations is faster than $\exp \left(-A t^{1 / 3}\right)$ may be related to similar behavior observed in experiments by Hunt et al. [13], who found that when temperature was lowered the measurements departed progressively from the expected behavior. This might be interpreted in the light of the present work because our simulations and calculations should approach the experimental re- sults as temperature is lowered, when the assumption that traps are quenched holds. However, from the available data it is not possible to make a definite conclusion on this point, because the experiments were not designed to clarify this specific question, and further work in this direction is needed to establish the relevance of both theory and simulation to actual physical properties. We envisage that, as simulation and experiment correspond to the same ordering in the $N \rightarrow \infty$ and $t \rightarrow \infty$ limits, their conclusions will be comparable. If, as we are confident about, this turns out to be the case, the theoretical analysis of diffusion problems in the presence of traps should be reexamined with the present conclusions in mind, and the contributions of rare events to situations where the traps are not perfect absorbers or there is spatial correlation between their location, to name just two examples, should be identified, thus establishing their relevance. We expect to pursue further this goal after new experiments or specifically designed, more precise simulations pave the way to a verification of these conclusions.

\section{Acknowledgement}

A.S. thanks the Universidad de Cantabria for hospitality during a stay when part of this work was done. Work at Madrid and Leganés is supported by CICYT under project MAT95-0325. Work at Cantabria is supported by DGICYT under project PB93-0054-C02-02.

\section{References}

[1] S. Alexander, J. Bernasconi, W.R. Schneider and R. Orbach, Rev. Mod. Phys. 53 (1981) 175;

J.W. Haus and K.W. Kehr, Phys. Rep. 150 (1987) 263;

S. Havlin and D. Ben-Avraham, Adv. Phys. 36 (1987) 695.

[2] V. Halpern and S. Shatz, Chem. Phys. 91 (1984) 233; A. Blumen and J. Klafter, J. Luminesc. 34 (1985) 77.

[3] P. Grassberger and I. Procaccia, J. Chem. Phys. 77 (1982) 6281 .

[4] B. Movaghar, G.W. Sauer and D. Würtz, J. Stat. Phys. 27 (1982) 473

[5] G.H. Weiss and S. Havlin, J. Stat. Phys. 37 (1984) 17

[6] L. Skala and O. Bilek, Phys. Lett. A 125 (1987) 262.

[7] Xian-Geng Zhao and Ji-Xing Liu, Phys. Lett. A 168 (1992) 165.

[8] Xian-Geng Zhao and Ji-Xing Liu, J. Phys. Condens. Matter 5 (1993) 4443.

[9] A. Sánchez, F. Domínguez-Adame and E. Maciá, Phys. Rev. B 51 (1995) 173. 
[10] F. Domínguez-Adame, B. Méndez, A. Sánchez and E. Maciá, Phys. Rev. B 49 (1994) 3839;

F. Domínguez-Adame, E. Maciá and A. Sánchez, Phys. Rev. B 50 (1994) 6453.

[11] P.A. Parris, Phys. Lett. A 114 (1986) 250.
[12] G.H. Weiss and R.J. Rubin, Adv. Chem. Phys. 52 (1983) 363, Fig. 6 on p. 423.

[13] I.G. Hunt, D. Bloor and B. Movaghar, J. Phys. C 16 (1983) L523; 18 (1985) 3497. 